\title{
NUEVOS SISTEMAS TERAPÉUTICOS DE AGENTES NEUROPROTECTORES EN EL TRATAMIENTO DEL GLAUCOMA
}

\section{NEW THERAPEUTIC SYSTEMS OF NEUROPROTECTORS AGENTS IN THE TREATMENT OF GLAUCOMA}

\author{
BRAO-OSUNA I ${ }^{1}$, CHECA P ${ }^{1}$, HERRERO-VANRELL $\mathrm{R}^{1}$
}

El glaucoma es una neuropatía degenerativa que constituye una de las primeras causas de ceguera en los países desarrollados. Aunque la patogénesis de esta enfermedad se desconoce, sí se asocia a una muerte gradual de las células ganglionares de la retina (RGC). Entre los factores que favorecen esta muerte celular, el aumento de la presión intraocular (PIO) ha sido considerado tradicionalmente como una causa esencial. Al aumentar la PIO, el suministro sanguíneo que llega a la retina se ve comprometido por el exceso de presión, viéndose afectado el tejido neuronal. Parece también que, al aumentar la presión sobre el tejido conectivo de la cabeza del nervio óptico (lámina cribosa), se interrumpe el flujo axoplasmático, bloqueando la llegada de factores neurotróficos endógenos al cuerpo neuronal desde los axones. Ante estas carencias, las células pueden desencadenar un proceso natural de degeneración celular (apoptosis). Actualmente se considera que la presión intraocular es el principal factor de riesgo para el desarrollo del glaucoma, si bien este puede desarrollarse con cifras de presión dentro de la normalidad estadística. De hecho, los medicamentos existentes en el mercado para el tratamiento del glaucoma están basados en la reducción y control de esta presión ocular. Sin embargo es importante destacar que ni todos los pacientes con PIO elevada desencadenan glaucoma ni la disminución de esta PIO asegura la protección contra la misma. Así, en la última década se están estudiando otros factores importantes en la génesis y en el proceso de la degeneración neuronal retiniana, como la influencia de la falta de aporte sanguíneo suficiente a la cabeza del nervio óptico (en ausencia de PIO elevada) y las células retinales adyacentes (1).
Recientes estudios en el campo de la fisiología celular han podido demostrar que el proceso de neurodegeneración puede describirse, cronológicamente hablando, en tres pasos: daño axonal primario; muerte de la neurona dañada, y daño y posterior muerte de neuronas adyacentes, en lo que se denomina «degeneración secundaria». Esta degeneración secundaria ocurre pues en neuronas inicialmente no dañadas pero que acaban muriendo por exposición a agentes citotóxicos liberados por la muerte de las neuronas con daño axonal primario (1). Entre los agentes citotóxicos se sabe, por ejemplo, que altas concentraciones de glutamato hiperestimulan sus receptores aumentando la concentración de calcio intracelular y desencadenando la producción de radicales libres que son altamente citotóxicos y producen la muerte neuronal. Basándose en estos hechos, la neuroprotección se centra en la protección de neuronas susceptibles de sufrir «degeneración secundaria». Esta neuroprotección es útil incluso cuando se desconoce la causa inicial de la enfermedad y tiene como objetivo limitar y prevenir el daño y muerte neuronal mediante el bloqueo de los mecanismos que la desencadenan (1).

El gran interés despertado por el uso de terapia neuroprotectora en el tratamiento del glaucoma en los últimos años se ha materializado en el creciente número de investigadores dedicados a este campo. La neuroprotección resulta una vía alternativa a la terapia basada en el control de la PIO, a veces insuficiente en muchas patologías glaucomatosas. Además, el desarrollo y los buenos resultados obtenidos en el tratamiento de otras alteraciones neuronales, como enfermedad de Huntington, enfermedad de

\footnotetext{
1 Doctora en Medicina. Departamento de Farmacia y Tecnología Farmacéutica. Universidad Complutense. Madrid. España.

E-mail: ibravo@farm.ucm.es / pcheca@farm.ucm.es / rociohv@farm.ucm.es
} 
Parkinson, enfermedad de Alzheimer, etc., auguran buenos presagios para el uso de esta nueva estrategia en el tratamiento del glaucoma (1).

Hasta el momento se sabe que la protección de las RGC se puede llevar a cabo desde varios frentes:

1. Prevención de la excitotoxicidad de RGC inducida por glutamato (antagonistas de los receptores NMDA (N-methyl-D-aspartate) como la memantina).

2. Bloqueo de las vías de apoptosis ( inhibidores de semaforina y caspasa).

3. Administración de factores neurotróficos (GDNF, BDNF, CNTF, T-588) .

4. Adminstración de secuestradores de radicales libres (Carnitina, Carnosina, coenzima Q10, ácidos grasos $\Omega 3$, Vitamina $B_{12}$, etc.).

5. Administración de bloqueantes de canales de calcio.

Estudios preliminares, llevados a cabo con algunas de estas moléculas en animales de experimentación, han ofrecido resultados prometedores en la protección de RGC $(2,3)$. Sin embargo, a diferencia del tratamiento para disminuir la PIO, que actúa sobre el segmento anterior del ojo y puede ser aplicado por vía tópica, la aplicabilidad de terapias neuroprotectoras pasa por desarrollar formas de administración que sean eficaces directamente a nivel de retina. En este sentido, la administración intravítrea parece la más directa y la que con mayor certeza puede controlar la cantidad de fármaco que accede a su lugar de acción, eliminando la mayor parte de los efectos secundarios sistémicos (4). No obstante, esta ruta no esta exenta de problemas. Además de los riesgos inherentes a la utilización de una técnica invasiva (infecciones, etc.), otro inconveniente de esta vía es que la administración del fármaco se realiza en forma de «bolus», lo que resulta insuficiente para el tratamiento efectivo de problemas crónicos como el glaucoma, que precisan concentraciones del agente activo en el lugar de acción mantenidas durante un largo periodo de tiempo.

Una vez descartada la administración repetida de inyecciones intravítreas, dadas las complicaciones derivadas, tales como hemorragias vítreas, desprendimiento de retina, cataratas, endoftalmitis, etc., se hace patente la necesidad de formas de administración intravítreas capaces de proporcionar concentraciones terapéuticas del fármaco en su lugar de acción por periodos prolongados de tiempo disminuyendo, en todo lo posible, el número de aplica- ciones. Los sistemas microparticulares biodegradables de liberación controlada se están convirtiendo en una alternativa muy interesante en el desarrollo de formulaciones intravítreas destinadas a este fin. Por un lado, estos sistemas pueden ser inyectados en forma de suspensión en el vítreo, sin necesidad de cirugía, y por otro lado, como se van degradando con el tiempo hasta desaparecer del lugar de acción, tampoco se necesita cirugía para su eliminación. Además, como van liberando progresivamente el fármaco, se pueden administrar dosis elevadas, lo que reduce considerablemente el número de aplicaciones, espaciándolas incluso varios meses. Así por ejemplo, varios autores trabajan en la microencapsulación de factores neurotróficos (BDNF, GDNF) en microsferas de quitosano o de ácido poli(láctico-co-glicólico) (PLGA) $(3,5-7)$ con resultados prometedores.

Finalmente otra vía alternativa, aún poco explorada en la administración de fármacos que tienen que actuar en el segmento posterior, es la administración por vía periocular (inyecciones subconjuntivales, subtenionianas, etc.). El fármaco administrado por esta vía debe atravesar la esclera, pudiendo alcanzar concentraciones terapéuticas en el vítreo y en la retina evitando las inyecciones intravítreas. En este sentido, las micro y nanopartículas con características superficiales capaces de aumentar la permeabilidad de fármacos a través de la esclera, podrían ser de gran utilidad en el tratamiento de enfermedades del segmento posterior del ojo, y en concreto del glaucoma.

A diferencia de otras enfermedades neurodegenerativas, el uso de neuroprotectores en el tratamiento del glaucoma esta muy poco desarrollado, sin que exista actualmente ninguna formulación en el mercado basada en neuroprotección. Es necesario, por tanto, un esfuerzo común por parte de los centros de investigación básica, hospitales, administraciones públicas, empresas farmacéuticas, etc., para poner lo antes posible al servicio de los pacientes y de los profesionales de la salud, el gran beneficio que supondría esta nueva estrategia terapéutica.

\section{BIBLIOGRAFÍA}

1. Ritch R. Complementary therapy for the treatment of glaucoma: a perspective. Ophthalmol Clin North Am 2005; 18: 597-609.

2. Garcia M, Forster V, Hicks D, Vecino E. Effects of muller glia on cell survival and neuritogenesis in adult porcine 
retina in vitro. Invest Ophthalmol Vis Sci 2002; 43: 3735 3743.

3. Mittal S, Cohen A, Maysinger D. In vitro effects of brain derived neurotrophic factor released from microspheres. Neuroreport 1994; 5: 2577-2582.

4. Ruiz-Moreno JM, Montero JA, Bayon A, Rueda J, Vidal M. Retinal toxicity of intravitreal triamcinolone acetonide at high doses in the rabbit. Exp Eye Res 2007; 84: 342348 .

5. Aubert-Pouessel A, Venier-Julienne MC, Clavreul A, Sergent $M$, Jollivet $C$, Montero-Menei $C N$, et al. In vitro study of GDNF release from biodegradable PLGA microspheres. J Control Release 2004; 95: 463-475.

6. Andrieu-Soler C, Aubert-Pouessel A, Doat M, Picaud S, Halhal M, Simonutti $M$, et al. Intravitreous injection of PLGA microspheres encapsulating GDNF promotes the survival of photoreceptors in the rdl/rdl mouse. Mol Vis 2005; 11: 1002-1011.

7. Ward MS, Khoobehi A, Lavik EB, Langer R, Young MJ. Neuroprotection of retinal ganglion cells in DBA/2J mice whit GDNF loaded biodegradable microspheres. J Pharm Sci 2006: 96: 558-568. 